\title{
REVIEW ARTICLE OPEN First principles calculation of spin-related quantities for point defect qubit research
}

\author{
Viktor Ivády $\mathbb{D}^{1,2}$, Igor A. Abrikosov ${ }^{1,3}$ and Adam Gali $\mathbb{D}^{2,4}$
}

Point defect research in semiconductors has gained remarkable new momentum due to the identification of special point defects that can implement qubits and single photon emitters with unique characteristics. Indeed, these implementations are among the few alternatives for quantum technologies that may operate even at room temperature, and therefore discoveries and characterization of novel point defects may highly facilitate future solid state quantum technologies. First principles calculations play an important role in point defect research, since they provide a direct, extended insight into the formation of the defect states. In the last decades, considerable efforts have been made to calculate spin-dependent properties of point defects from first principles. The developed methods have already demonstrated their essential role in quantitative understanding of the physics and application of point defect qubits. Here, we review and discuss accuracy aspects of these novel ab initio methods and report on their most relevant applications for existing point defect qubits in semiconductors. We pay attention to the advantages and limitations of the methodological solutions and highlight additional developments that are expected in the near future. Moreover, we discuss the opportunity of a systematic search for potential point defect qubits, as well as the possible development of predictive spin dynamic simulations facilitated by ab initio calculations of spin-dependent quantities.

npj Computational Materials (2018)4:76; https://doi.org/10.1038/s41524-018-0132-5

\section{INTRODUCTION}

In the last decades considerable efforts have been made to utilize fundamental aspects of quantum mechanics in various visionary applications, such as quantum information processing and quantum computation. ${ }^{1}$ The conceptual building block of these revolutionary applications is the quantum bit (qubit), the simplest possible quantum system that includes only two levels. In practice, the levels implementing the qubit states must be isolated from the environmental degrees of freedom and at the same time they must remain controllable via different external means. Simultaneous fulfillment of these criteria causes one of the major challenges in qubit implementations.

Qubits have been already demonstrated in a broad range of physical systems. ${ }^{1}$ Point defect-based quantum bits in semiconductors are among the most recent realizations, ${ }^{2-7}$ where generally the spin of a paramagnetic point defect or associated paramagnetic nuclei gives rise to the quantum states that may be manipulated by electric and magnetic fields, microwave irradiations, and optical means. ${ }^{3}$ Due to the special characteristics of the point defect qubits and the properties of the semiconducting host material, the existing point defect quantum bits are well isolated from their environment., ${ }^{3,7}$ This leads to observations of generally long coherence times $\mathrm{s}^{3,8}$ and qubit operation even at room temperature. ${ }^{9-11}$ For example, in isotope engineered high-purity diamond sample the coherence time can exceed a millisecond at room temperature. ${ }^{9}$ These attributes together with the possibility of magneto-optical control make point defect qubits, including single or ensemble of isolated qubits, highly promising for numerous applications. For instance, point defect qubits created in nanocrystals or close to the surface of nano-fabricated thin film samples can be utilized as atomic-scale temperature, ${ }^{12}$ electric field, ${ }^{13}$ and strain $^{14}$ sensors, as well as magnetic resonance probes $^{15-17}$ that are about to revolutionize nanoscale metrology. Ensembles of point defects qubits in larger samples can be used to greatly increase the sensitivity of microscale room temperature sensors $^{18,19}$ and gyroscopes ${ }^{20,21}$ with some of them readily integrable in existing semiconducting electric devices. Quantum optics devices are intensively studied for quantum information processing applications $s^{22,23}$ and for testing fundamental aspects of quantum entanglement. Indeed, the loophole-free Bell test was first demonstrated by point defect qubits. ${ }^{24}$ Silicon-based quantum computation maybe be realized by using single point defect spins and quantum dots. ${ }^{1,5,25}$ Furthermore, point defect qubits can be used to polarize nearby nuclear spins, ${ }^{26,27}$ thus ensembles of these nuclear polarization sources can lead to the hyperpolarization of the host material that may be utilized in nuclear magnetic resonance and imaging to enhance sensitivity by orders of magnitudes. ${ }^{28}$

The quality of the point defect qubits, i.e. the fidelity of spin state manipulation and read-out and the decoupling from the environment degrees of freedom, determines the capabilities and limitations of the potential applications. The quality of the point defect qubits relies on the other hand on the properties of the semiconductor host material and the point defect that give rise to the isolated electron or nuclear spin states. Consequently, understanding and engineering point defect qubits and their applications often translate to material science and material engineering problems. Considering the vast diversity of these

\footnotetext{
${ }^{1}$ Department of Physics, Chemistry and Biology, Linköping University, SE-581 83 Linköping, Sweden; ${ }^{2}$ Wigner Research Centre for Physics, Hungarian Academy of Sciences,

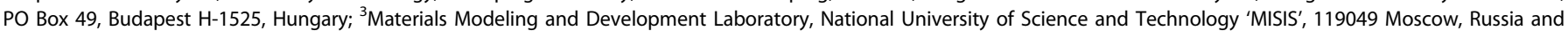
${ }^{4}$ Department of Atomic Physics, Budapest University of Technology and Economics, Budafoki út 8, Budapest H-1111, Hungary Correspondence: Viktor Ivády (ivady.viktor@wigner.mta.hu)
} 
fields, there are many potential directions for future point defect qubit research.

In this respect, computational studies play an essential role in material science because of the detailed physical picture they provide and due to their significant predictive power. First principles electronic structure calculations have greatly contributed to the quantitative understanding of point defect qubitrelated materials, leading to the fast development of the field. On the other hand, the emerging research directions generally require the development of novel computational methods and tools. Specifically, point defect qubit studies require high precision electronic structure calculations of ground and excited state properties, as well as all kinds of derived spin-dependent properties at various environmental conditions.

Here, we report on recent developments and applications of first principles calculations of point defects electronic structure and spin-related quantities for solid-state qubits in semiconductors. By going through the recent achievements in this area, we highlight required additional developments and point out possible new directions that can further increase the predictive power and area of applications of the computational methods.

\section{PHYSICS OF POINT DEFECT QUBITS}

There are two major classes of point defect qubits in semiconductors. In the first type of qubits, electron and nuclear spins of shallow donors, such as phosphor and bismuth, in silicon are manipulated through electrical-gates (quantum dots). 5,8,25,29,30 Spin-orbit and hyperfine interactions play a crucial role in the physics and applications of these qubits. The corresponding spindependent quantities can be calculated from first principles as we discuss in Section 4.

In this section, we focus on the second class of point defect qubits, i.e. deep-level high spin state color centers in wide band gap semiconductors whose spin can be manipulated by optical means. Using confocal microscopy techniques, it is possible to excite and collect photons from defects in a volume of $\mathcal{O}\left(10 \mu \mathrm{m}^{3}\right)$ of a semiconducting sample. ${ }^{31}$ For low point defect concentration the examined volume may contain only a few, ultimately one single color center. Individual color centers in semiconductors that exhibit fast bound-to-bound optical transition may implement single photon emitters that often operate even at room temperature. ${ }^{22,32-34}$ Optically controllable point defect qubits form a special class of single photon emitters. Due to this duality, point defect qubits exhibit great potential in a broad range of quantum information processing and sensing applications. ${ }^{2,3,7,35}$

For qubit implementation, a color center must fulfill additional requirements: it must form high spin ground and/or excited state, the electron spin must exhibit long coherence time, and the luminescence of the defect should be spin dependent. The latter requirement enables optical initialization and read-out of a point defect qubit. Additionally, applying external magnetic field and microwave irradiation, full control of the point defect spin states can be achieved.

The most thoroughly investigated optically active point defect qubits are the negatively charged nitrogen substitutional-vacancy complex in diamond (NV center), $, 936,37$ the neutral silicon-carbon double vacancy in SiC polytypes (divacancy), ${ }^{10,38}$ and the isolated negatively charged silicon vacancy in SiC polytypes (silicon vacancy). ${ }^{11,39}$ Additionally to these well-established qubits, there are several emerging qubits and qubit candidates in diamond and in other semiconductors. Group IV substitutional-vacancy complex in diamond, such as SiV center, ${ }^{40,41} \mathrm{GeV}$ center, ${ }^{42}$ and SnV center ${ }^{43}$ chalcogen double donors in silicon ${ }^{44}$ (for example $S^{+}$ and $\mathrm{Se}^{+}$), and read-earth ions in optical materials, like $\mathrm{Ce}^{3+}$ in yttrium aluminum garnet ${ }^{45}$ (YAG), $\mathrm{Pr}^{3+}$ ions in yttrium orthosilicate $^{46}$ (YSO), are among the most recently investigated qubits and qubit candidates. Furthermore, we note that phosphor donorrelated optical transition in silicon was recently utilized to achieve advanced control of ${ }^{31} \mathrm{P}$ nuclear spin states. ${ }^{47}$ For further reading see the recent review by Awschalom et al. ${ }^{48}$

In the following, we discuss NV center, divacancy, and silicon vacancy room temperature qubits in more details. The polytypic $\mathrm{SiC}$ host often gives rise to symmetrically non-equivalent configurations of a considered point defect. Consequently, divacancy and silicon vacancy qubits represent families of several distinguishable configurations with slightly different characteristics and varying potential for applications rather than a single qubit. $^{49}$

NV center and divacancy exhibit qualitatively similar electronic structure, $^{50}$ therefore we discuss the physics of these defects together. The four (six) dangling bonds of the NV center (divacancy) form two $a_{1}$ and one $e$ (two $a_{1}$ and two e) single particle states that are occupied by six (six) electrons. According to $\mathrm{ab}$ initio density functional theory calculations (DFT), ${ }^{50-53}$ a fully occupied lower lying $a_{1}$ state and an $e$ state, occupied by two electrons, appear in the lower part of the band gap for both defects (see Fig. 1b-d). In the case of divacancy, an empty e state can also be found close to the conduction band edge, however, it has negligible effect on the properties of the defect. Due to the half occupied $e$ state above the $a_{1}$ state, both defects exhibit a spin-1 ground state of ${ }^{3} \mathrm{~A}_{2}$ symmetry, see Fig. 2a for the NV center. Relativistic effects, mostly dominated by the dipolar coupling of the unpaired electron spins, split the substates of the triplet ground states (see Fig. 2a), by $\approx 2.9$ and $\approx 1.3 \mathrm{GHz}$ for the NV center $^{54}$ and divacancy, ${ }^{55}$ respectively. Spin conserving optical excitation can drive the system into the lowest optically exited state of ${ }^{3} \mathrm{E}$ symmetry. In single particle picture, an electron is promoted from the $a_{1}$ state to the lowest $e$ state (see Fig. $1 \mathrm{~b}-\mathrm{d}$ ). Note that this electronic configuration gives rise to a dynamic a Spin density of the b NV center NV center

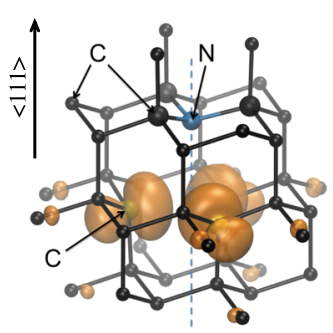

\section{Diamond}

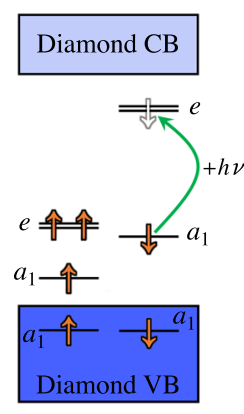

c Divacancy

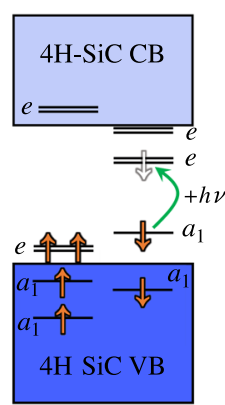

d Silicon vacancy

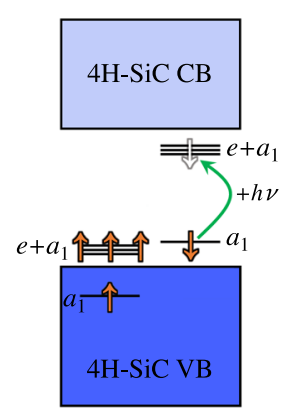

Fig. 1 a Structure and spin density of the NV center in diamond, and $\mathbf{b}$-d Kohn-Sham electronic structure of the NV center in diamond and divacancy and silicon vacancy in $4 \mathrm{H}-\mathrm{SiC}$, respectively. Green arrows represent the lowest energy optical transition in the single particle picture. The Kohn-Sham electronic structures were obtained by convergent HSE06 hybrid functional calculations (data from refs ${ }^{52,53,65}$ ) 
Jahn-Teller system with strong electron-phonon interaction and effective $C_{3 v}$ symmetry when the axis of the pair defect is parallel to the high symmetry axis of the host material. Due to the spin-orbit and spin-spin dipolar interactions, the ${ }^{3} E$ state exhibits a complicated fine structure at low temperature. ${ }^{56-58}$ At higher temperature, the spin-orbit interaction and off-axis components of the spin-spin zero-field-spitting interaction average out due to the motion of the atoms. ${ }^{59}$ The high-temperature fine structure of the ${ }^{3} \mathrm{E}$ state resembles the fine structure of the ground state, i.e. it can be parameterized by the axial zero-field-splitting parameter D. ${ }^{59}$

Due to the different occupation of the dangling bonds, the energy minima of the ground and the optically excited states belong to two different atomic configurations in the configuration space (see Fig. 3). As a consequence, phonons play an important role in optical excitation and luminescence processes. Optical excitation from the ground state most probably ends up in a vibronic excited state. The probability of the transition proportional to the overlap of the ground and the vibronic excited states. After excitation, the vibronic excited state quickly relaxes by emitting phonons. A similar mechanism takes place during the luminescence processes. Direct transition between the nophonon-excited ground and excited states, that give rise to the so-called zero-phonon luminescence ( $\mathrm{ZPL}$ ) and absorption lines, is allowed by the zero-point motion of local vibrational modes. The energy difference of the most probable phonon-assisted absorption and the ZPL transition is the Stokes shift, while the energy difference of the most probable phonon-assisted emission and the ZPL is the anti-Stokes shift.

a

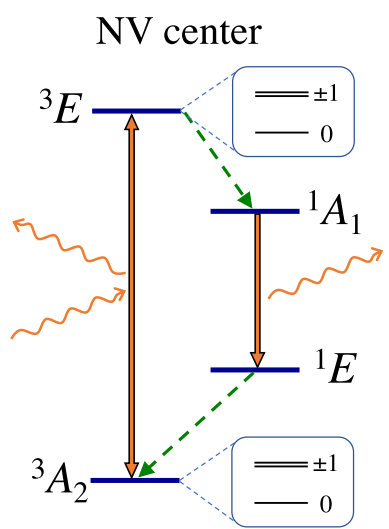

b

\section{Silicon vacancy}

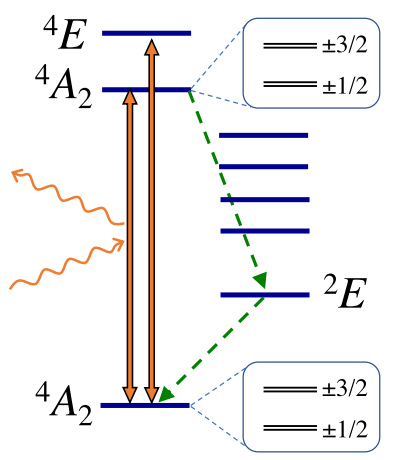

Fig. 2 Low energy many particle spectrum of (a) the NV center in diamond (discussed in refs ${ }^{60,61}$ ) and (b) silicon vacancy in SiC (discussed in ref. ${ }^{67}$ )
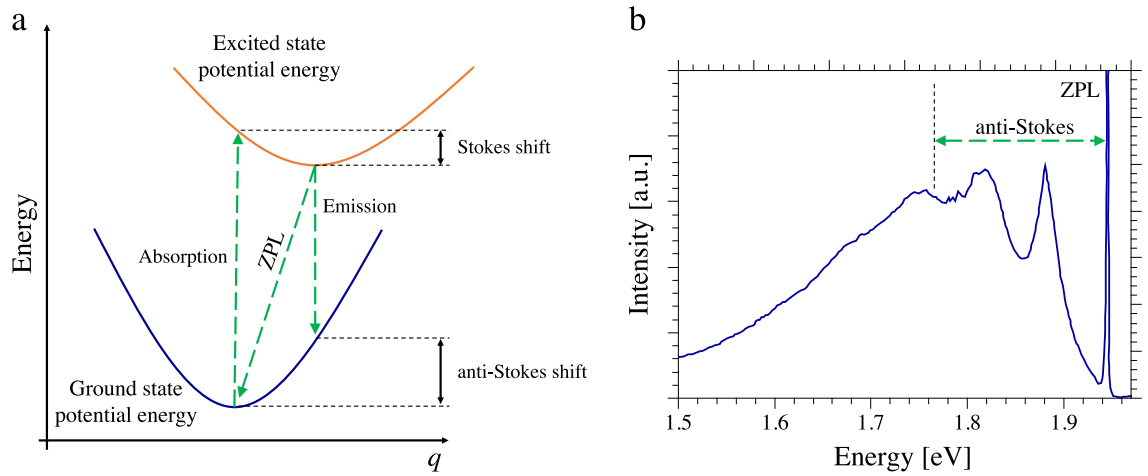

Fig. 3 a Schematic diagram of the configuration coordinate diagram of a point defect's ground and excited states. Green arrows show the most relevant phonon-assisted and zero-phonon (ZPL) optical transitions. b Luminescence spectrum of the NV center (data from ref. ${ }^{158}$ ) 
responsible for spin relaxation mechanism in these qubit implementations. Purification of the host material from paramagnetic isotopes and point defects were successfully used to elongate relaxation and coherence times. ${ }^{9,38}$

\section{FIRST PRINCIPLES ELECTRONIC STRUCTURE CALCULATIONS}

Theoretical characterization of point defects qubits and first principles calculations of spin coupling parametes depend crucially on the outcomes of the underlying electronic structure calculations. Therefore, it is essential to understand the capabilities and limitations of the latter before we discuss the results of the calculations. Unfortunately, due to the large structural model, which is generally needed for the accurate description of single point defects in bulk semiconductor hosts, one must always compromise between the accuracy and computational efficiency. DFT is the most widely used method in the field. However, there are numerous attempts to use more sophisticated Green's function and wavefunction-based methods for point defect qubit calculations, see later in this section.

Let us therefore shortly review the most relevant electronic structure theories used in ground and excited state calculations of point defect qubits. As a first basic approximation, one generally assumes that the wavefunctions of the nuclei and the electrons can be separated (the Born-Oppenheimer approximation). Relying on this approximation, the electronic structure problem can be solved for every fixed arrangement of the atomic nuclei. Note, however, that when the point defect qubit has Jahn-Teller unstable state(s), for instance, the excited state of the NV center and divacancy, one must go beyond the Born-Oppenheimer approximation (see Section 4).

Ground state methods

DFT $^{69,70}$ in the Kohn-Sham ${ }^{71,72}$ and generalized Kohn-Sham ${ }^{73}$ formalisms are the most frequently used methods for the ground state electronic structure calculation of cluster or supercell models of point defects in semiconductors. In these two formalisms, the Schrödinger problem of many-electron system is mapped onto the problem of auxiliary non-interacting and partially interacting Kohn-Sham particles, respectively. The mapping is constructed so that the auxiliary system reproduces the one particle density and the total energy of the considered system. ${ }^{70,72}$ Exact calculations require the definition and calculation of the exchange-correlation energy term as a functional of the density. The exact definition of the exchange-correlation energy functional is not known in general, thus it is approximated in practice. ${ }^{70,72}$

In first principles studies of point defects qubits in semiconductors, the most widely used approximate exchange-correlation functionals are the $\mathrm{PBE}^{74}$ functional and the HSE06 ${ }^{75,76}$ hybrid functional. The latter functional models a partially interacting system in the framework of generalized Kohn-Sham scheme. ${ }^{73}$ HSE06 hybrid functional includes the calculation of the exact exchange energy of the Kohn-Sham particles, ${ }^{75,76}$ which makes this functional computationally more demanding. In conventional semiconducting hosts, HSE06 hybrid functional provides the most accurate results for bulk and $s p$-point defect-related quantities in general, ${ }^{77-79}$ as we discuss later in this paper. There are two main reasons for the improved performance of HSE06: (1) it exhibits a derivative discontinuity in the exchange correlation potential ${ }^{80}$ that remedies the band gap underestimation problem of semilocal Kohn-Sham DFT functionals in conventional semiconductors $^{77,81}$ and (2) localized defect states often satisfy the generalized Koopman's theorem ${ }^{78,82}$ thus there is no self-interaction for the auxiliary particles occupying the defect states. ${ }^{83}$

We note that hybrid functionals may provide quantitatively less accurate $^{84-86}$ and even qualitatively incorrect results ${ }^{85,87,88}$ for electronic structures of varying electron localization. It is well established that the exact exchange-semilocal exchange mixing parameters must be reconsidered when hybrid functionals are applied to semiconducting transition metal compounds. ${ }^{84}$ The need for different exact exchange ratio for states of different degree of localization can also alter the calculation of transition metal impurity-related point defects in conventional semiconductors of $s p$ hybridized valence and conduction bands. ${ }^{85}$ Therefore, additional considerations are needed to find a reliable hybrid functional when $d$ and $f$ orbitals play a role. ${ }^{84,89}$

Supercell structural models of point defects with periodic boundary conditions are the most often used approaches to investigate point defect qubits in semiconductors. In such models, plane wave basis set is the most suitable for expressing Kohn-Sham single particle wavefunctions in the inter-atomic region. Interaction with the atomic cores, including the potential of the nuclei and the core electrons, is described either by pseudopotentials or by the projector augmented wave (PAW) method $^{90}$ in most of the calculations. The latter enables the calculation of atomic core-related quantities, such as spin-orbit interaction and hyperfine Fermi contact term with all-electron accuracy, see later in Section 4.

Convergence and numerical accuracy are always important aspects of first principles DFT calculations. The most relevant technical parameters that determine the accuracy of the calculations are the supercell size, Brillouin zone sampling, basis set, and the force or energy criteria used for the structural optimization. As will be shown in Section 4, in the case of spin coupling parameter calculations, often very high numerical accuracy is needed. Recently, thorough numerical tests, including ground state, optical, and hyperfine parameters calculations were carried out for the divacancy qubit in $4 \mathrm{H}-\mathrm{SiC}$ in refs 53,91 .

From the ground state spin density and Kohn-Sham particle wavefunctions most of the relevant ground state spin-dependent properties can be approximately calculated, see Section 4.

\section{Excited state methods}

As we have seen in Section 2, spin state initialization and read-out can be implemented through the optical cycle of point defect qubits. This process is determined by the electronic structure of the point defects, especially, on the excited state spectrum and the coupling and inter-system-crossing of different states. There are several wave-function-based quantum chemistry approaches that can provide such spectrum from first principles, however, majority of these methods are applicable only for small systems. Large point defect models with the periodic boundary conditions are challenging for such calculations. The most widely used approaches today apply computationally less demanding DFTbased methods, for instance, the constrained occupation DFT method. Limited accuracy of this method however motivated development of efficient, still more advanced computational approaches.

Constrained occupation DFT. In constrained occupation DFT, ${ }^{92}$ the total energy of a point defect excited state configuration is approximated by the DFT total energy of a Kohn-Sham system, where one of the occupied Kohn-Sham defect states is depopulated, while a higher lying unoccupied Kohn-Sham state is populated (see Fig. $1 b-d$ ). The constrained occupation of the orbitals is kept fixed during the self-consistent solution of the Kohn-Sham equations. Note that structural optimization of the systems is still possible within this approach as the total energy is well-defined. Consequently, besides the absorption energy, which requires the same ground and excited state atomic configuration in the Franck-Condon approximation, the emission and zerophonon energy, as well as the Stokes and anti-Stokes shifts can be calculated in the constrained occupation DFT (see Fig. 3). In Table 1 , one can see these quantities for the NV center. Remarkable 
Table 1. Optical properties of the NV center from first principles constrained occupation DFT

\begin{tabular}{llll}
\hline & PBE & HSE06 & Exp. \\
\hline Absorption energy & 1.910 & 2.213 & 2.180 \\
Zero-phonon line energy & 1.706 & 1.955 & 1.945 \\
Emission energy & 1.534 & 1.738 & 1.760 \\
Stokes shift & 0.204 & 0.258 & 0.235 \\
Anti-Stokes shift & 0.172 & 0.217 & 0.185 \\
\hline
\end{tabular}

Theoretical values were calculated by PBE and HSE06 functionals in ref. ${ }^{92}$ Experimental values are reported in ref. ${ }^{154}$

accuracy was reported for the optical properties calculated by HSE06 functional. ${ }^{92}$ For more details on first principles calculation of optical properties see ref. ${ }^{93}$

The constrained occupation DFT inherently assumes that the Kohn-Sham orbitals of the defect states have a true physical meaning, thus the change of the occupancies can indeed mimic a realistic excitation process. According to the generalized Koopmans' theorem, ${ }^{94,95}$ or in other context the ionization potential theorem, ${ }^{80,96}$ this is only fulfilled for the highest occupied state when its Kohn-Sham single particle energy is equal to the negative of the ionization potential,

$\varepsilon_{\mathrm{ho}}+\Delta \varepsilon_{\mathrm{cc}}=-\left(E_{N}-E_{N-1}+\Delta E_{\mathrm{cc}}\right)$,

where $\varepsilon_{\text {ho }}$ is the energy of the highest occupied Kohn-Sham orbital and $E_{N}$ is the total energy of the $N$ electron system. Note that due to the periodic boundary condition, finite size effects can be strong for charged supercells, both in terms of the Kohn-Sham eigenenergies and the total energies, and they must be corrected by appropriate charge correction terms, $\Delta \varepsilon_{\mathrm{cc}}$ and $\Delta E_{\mathrm{cc}}$, respectively. ${ }^{97}$

Recent theoretical studies ${ }^{78,82}$ demonstrated that defect states described by HSEO6 functional often fulfill Eq. (1). Thus the constrained occupation DFT may describe realistic excitation processes, which explains the remarkable accuracy seen in Table 1.

The constrained occupation DFT describes excitation processes in terms of a single Slater determinant of the Kohn-Sham particles. The constraint occupation DFT method, however, often fails to provide accurate total energies of states that are highly correlated, or in the language of Hartree-Fock theory, are multideterminant in nature. Open shell singlet states fall into this category. This limitation greatly hinders the computational description of the spin selective non-radiative decay process that may include transition through open shell singlet states.

Excited state calculation beyond constrained occupation DFT. In order to calculate highly correlated states, several alternative methods have been proposed and tested for the NV center in diamond. Parameters of the excited state spectrum obtained by different first principles methods are summarized and compared with the experimental values in Table 2.

The most widely used method to describe excited state phenomena in solids is the GW approximation to the Hedin equations ${ }^{98}$ in the framework of many-body perturbation theory. The method can be combined with the colution of the Bethe-Salpeter equation (BSE) ${ }^{99}$ to include electron-hole interactions and to describe singlet states. GW+BSE method was applied in simulations of the NV center by Ma et al. in ref. ${ }^{100}$, where three singlet states, ${ }^{1} E,{ }^{1} A_{1}$, and ${ }^{1} E^{\prime}$ states, were found between the triplet ${ }^{3} \mathrm{E}$ excited and ${ }^{3} \mathrm{~A}_{2}$ ground states. This result is in contradiction with the currently accepted energy structure of the NV center discussed in Section 2. Furthermore, the calculated $0.59 \mathrm{eV} Z \mathrm{ZPL}$ energy of the ${ }^{1} \mathrm{~A}_{1} \rightarrow{ }^{1} \mathrm{E}$ transition is approximately half
Table 2. Parameters of the electronic structure of the NV center in diamond as calculated by different theoretical approaches

\begin{tabular}{|c|c|c|c|}
\hline Method & ${ }^{3} \mathrm{E} \rightarrow{ }^{3} \mathrm{~A}_{2}$ & ${ }^{1} \mathrm{~A}_{1} \rightarrow{ }^{1} \mathrm{E}$ & ${ }^{3} E \rightarrow{ }^{1} A_{1}$ \\
\hline$* \mathrm{GW}+\mathrm{BSE}^{100}$ & 2.09 & 0.59 & 1.10 \\
\hline $\mathrm{Cl}$ on $\mathrm{C}_{42} \mathrm{H}_{42} \mathrm{~N}^{101}$ & 1.93 & 1.43 & -0.1 \\
\hline Ext. Hubb. + DFT par. ${ }^{102}$ & 2.38 & 0.62 & 1.35 \\
\hline * Ext. Hubb. + GW fit. ${ }^{103}$ & 2.0 & 0.96 & 0.6 \\
\hline$* \mathrm{Cl}+\mathrm{CRPA}^{105}$ & 2.05 & 0.89 & 0.69 \\
\hline Experiment & $1.945^{154}$ & $1.19^{155}$ & $\approx 0.4^{132}$ \\
\hline
\end{tabular}

of the experimental value, suggesting that $\mathrm{G}_{0} \mathrm{~W}_{0}+\mathrm{BSE}$ method fails to accurately describe important static correlation effects in the singlet states.

Quantum Monte Carlo configuration interaction (QMC Cl) calculation on small cluster models ${ }^{101}$ and generalized Hubbard model-based approaches, ${ }^{102,103}$ on the other hand, are able to qualitatively reproduce the accepted energy level structure of the NV center, i.e. they predict only two shelving states that can be connected to the nonradiative decay process of the NV center (see Table 2).

In the QMC Cl approach on a $\mathrm{C}_{42} \mathrm{H}_{42} \mathrm{~N}^{-}$cluster, Delaney et al. ${ }^{101}$ reported that the higher lying ${ }^{1} \mathrm{~A}_{1}$ shelving state appears slightly above the ${ }^{3} E$ states. This result suggests higher energy inter system crossing and thus lower decay rate. Furthermore, the ${ }^{1} \mathrm{~A}_{1} \rightarrow{ }^{1} \mathrm{E}$ transition energy is overestimated (see Table 2). The discrepancies can most probably be attributed to the very small cluster model used in the calculations.

In the third-type of approaches extended Hubbard model Hamiltonians were diagonalized for the electrons occupying the dangling bonds of the NV center

$$
\begin{gathered}
H=\sum_{i j \sigma} t_{i j} c_{i \sigma}^{\dagger} c_{j \sigma} \\
+\sum_{i} U_{i} n_{i \uparrow} n_{i \downarrow} \\
+\frac{1}{2} \sum_{i \neq j, \sigma \sigma \prime} V_{i j} n_{i \sigma} n_{j \sigma \prime} \\
+\frac{1}{2} \sum_{i j l m, \sigma \sigma \prime} X_{i j l m} c_{i \sigma}^{\dagger} c_{j \sigma,}^{\dagger} c_{m \sigma \prime} c_{l \sigma},
\end{gathered}
$$

where $i, j, l$, and $m$ are dangling bond indexes, $\sigma$ and $\sigma^{\prime}$ are the spin indexes, $c, c^{\dagger}$, and $n^{\dagger}$ are the annihilation, creation, and number operators, $t$ is the hopping and ionic term, $U$ and $V$ are the intra and intersite Coulomb repulsion, and $X$ is the exchange interaction term. In ref. ${ }^{102}$, Ranjbar et al. calculated the hopping and interaction parameters from the defect orbitals obtained by DFT calculations with B3LYP functional ${ }^{104}$ on a $\mathrm{C}_{71} \mathrm{H}_{85}$ cluster. This method somewhat overestimates the ${ }^{3} \mathrm{E} \rightarrow{ }^{3} \mathrm{~A}_{2}$ transition energy, while substantially underestimates the ${ }^{1} A_{1} \rightarrow{ }^{1} E$ ZPL energy (see Table 2). In ref. ${ }^{103}$, Choi et al. applied a different approach to paramaterize a Hubbard Hamiltonian that neglected the exchange interaction term in Eq. (2). In order to determine $U$ and $V$, they compared quasi particle energies as obtained from GW calculation and from the diagonalization of the Hubbard Hamiltonian. Through this fitting, the screening effect of the delocalized semiconductor states was included to some extent. Despite omitting the exchange interaction term, this approach provides accurate excited state spectrum parameters.

In a very recent work, ${ }^{105}$ Bockstedte et al. combined constrained random phase approximation (CRPA) ${ }^{106}$ and $\mathrm{Cl}$ calculations to simultaneously include local static correlation effects of the dangling bonds and dynamic screening effects of the delocalized electronic states of the host semiconductor. In particular, $\mathrm{Cl}$ 
calculation was carried out on a small basis of hybrid-DFT Kohn-Sham orbitals that included both in-gap defect states and a few states at the valence and conduction band edges. Instead of the bare Coulomb potential, a screened potential, $V_{\text {eff }}=\varepsilon_{C R P A}^{-1} V$, was used. The CRPA dielectric function $\varepsilon_{\text {CRPA }}^{-1}$ was calculated by excluding transitions within the $\mathrm{Cl}$ basis in order to avoid double counting of correlation effects.

As can be seen in Table 2, accurate results can be achieved by the last two considered methods, i.e. by GW parameterized Hubbard Hamiltonian and $\mathrm{Cl}+\mathrm{CRPA}$ approaches. Both of these methods include static correlation effects via an appropriately screened interaction potential, indicating the importance of taking both effects into account.

\section{FIRST PRINCIPLES SPIN COUPLING PARAMETER CALCULATION}

Standard DFT calculations on supercell point defect models can achieve numerical accuracy as good as $1 \mathrm{meV}$. Even in this case, the energy scale of the spin coupling parameters can be orders of magnitude smaller (in the $\mu \mathrm{eV}$ range), which could make the impression that DFT would not be suitable for calculating these quantities. As we demonstrate in the following, it is not the case. Most of spin-related coupling parameters are small only due to the factors in front of the integrals of the Kohn-Sham particles. As the integral itself can be highly accurate, the relative numerical accuracy of the spin coupling parameters can be as good as the total energy.

\section{g-tensor}

Magnetic field is traditionally used to control the splitting of the spin states of point defect qubits, and vice versa, it is possible to detect magnetic field variations by measuring the splitting of the spin states. The spin Hamiltonian operator for defect spins with $S \leq 1$ and for negligible first-order ground state spin-orbit interaction can be written as

$$
\hat{H}_{\text {Zeeman }}=\mu_{\mathrm{B}} \mathbf{B g} \widehat{\mathbf{S}} \text {, }
$$

where $\mu_{\mathrm{B}}$ is the Bohr magneton, $\mathbf{B}$ is the magnetic field vector, $\widehat{\mathbf{S}}$ is the electron spin vector operator, and the $\mathbf{g}$ is the $g$-tensor that includes different higher order relativistic material dependent contributions to the $g_{e} g$-factor of the free electron. The Cartesian elements of the $g$-tensor can be obtained from the second derivative of the relativistic many electron energy $E$, as ${ }^{107}$

$$
g_{a b}=\left.\frac{1}{\mu_{\mathrm{B}}} \frac{\partial^{2} E}{\partial B_{a} \partial S_{b}}\right|_{B=0, S=0},
$$

which give rise to three additional non-negligible terms beside the $\hat{H}_{Z-\text { free }}$ Zeeman term of the free electron

$\hat{H}_{\text {Zeeman }}=\hat{H}_{Z \text {-free }}+\hat{H}_{Z-K E}+\hat{H}_{Z-\text { SO }}+\hat{H}_{Z-\text { SOO }}$,

where $\hat{H}_{Z-K E}$ is the electron Zeeman kinetic energy correction, and $\hat{H}_{Z-S O}$ and $\hat{H}_{Z-S O O}$ are the spin-orbit and spin-other-orbit corrections to the Zeeman energy. The definition of these terms can be found in the literature, for instance in refs ${ }^{107-109}$ The effective $g$-tensor of the system is defined as

$\mathbf{g}=g_{\mathrm{e}} \mathcal{I}+\Delta \mathbf{g}_{\mathrm{Z}-\mathrm{KE}}+\Delta \mathbf{g}_{\mathrm{Z}-\mathrm{SO}}+\Delta \mathbf{g}_{\mathrm{Z}-\mathrm{SOO}}$,

where $\mathcal{I}$ is the $3 \times 3$ identity matrix, and $\Delta \mathbf{g}_{z-x}$ are the corresponding correction tensors.

Implementations that are suitable for point defect $g$-tensor calculations in periodic models were provided by Sebastiani et al.

110 and Pickard et al. ${ }^{109}$ In the former approach pseudopotentials were used without correction terms in the core region that resulted in limited applicability of the method. Later, this limitations was relaxed by extending the method in ref. ${ }^{111}$ On the contrary, Pickard et al. ${ }^{109}$ used gauge including projector augmented wave approach ${ }^{112}$ (GIPAW), which allowed for all-
Table 3. Possible electron spin-electron spin and electron spin-magnetic field terms in the spin Hamiltonian ${ }^{116}$

\begin{tabular}{llllll}
\hline$S$ & Terms & & & & \\
\hline 0.5 & $\hat{S} B$ & & & & \\
1 & $\hat{S} B$ & $\hat{S}^{2}$ & & & \\
1.5 & $\hat{S} B$ & $\hat{S}^{2}$ & $\hat{S}^{3} B$ & & \\
2 & $\hat{S} B$ & $\hat{S}^{2}$ & $\hat{S}^{3} B$ & $\hat{S}^{4}$ & \\
2.5 & $\hat{S} B$ & $\hat{S}^{2}$ & $\hat{S}^{3} B$ & $\hat{S}^{4}$ & $\hat{S}^{5} B$ \\
\hline
\end{tabular}

Here, $\hat{S}$ represents any linear combination of the spin Cartesian operators, while $B$ represents any linear combination of the Cartesian magnetic field components

electron $g$-tensor calculations. Later, implementations using atomic orbitals in periodic boundary conditions were also presented. ${ }^{113} \mathrm{~g}$-tensor calculations were recently applied in the identification of the microscopic configuration of the nitrogen substitutional-silicon vacancy pair defect in different polytypes of $\mathrm{SiC}^{114}$ This new family of spin-1 point defects was recently suggested as a potential new platform for implementing point defect qubit in technologically mature $\mathrm{SiC}$ hosts. ${ }^{115}$

So far we considered only bi-linear terms in the Zeeman spin Hamiltonian. For defects with $S>1$, higher order magnetic field interaction terms are possible. ${ }^{108,116}$ In Table 3, we collected the possible higher order magnetic field interaction terms up to $S=5$ / 2. Note, that Table 3 does not contain higher order terms in $B$. Such terms are also possible, however, most often they can be safely neglected. ${ }^{116}$

Theoretical formulas for the higher order interaction terms can be found in the literature, ${ }^{116,117}$ however, less attention has been paid to the implementation and calculation of corresponding higher order $g$-tensor parameters so far. On the other hand, in recent experiment on the spin-3/2 silicon vacancy qubits in $4 \mathrm{H}-\mathrm{SiC}$ forbidden electron spin transitions were observed in the ODMR spectrum. ${ }^{118}$ To explain this result, higher order $\hat{S}^{3} B$ like terms had to be taken in the considerations. The $g$-tensor elements corresponding to these third-order terms were found to be considerable, i.e. $g_{3 \perp}+g_{3 \|} \approx 1.0$ and $g_{3 \perp}-g_{3 \|} \approx 0.2$. These results indicated that implementations of higher order magnetic field interaction term calculation may be desirable in the future to understand forbidden transitions.

\section{Spin-spin contribution to zero-field splitting}

Due to intra-defect interactions, the spin sublevels of the point defect qubits may split even at zero magnetic field. There are two major contributions to the, so-called, zero-field-splitting (ZFS): the spin-spin and the spin-orbit dipole interactions. In this section, we discuss first principles calculations of the former interaction, which is generally the most relevant interaction in the ground state of the considered solid state qubits.

When a point defect has more than one unpaired electron, each pair of the electrons spins $\widehat{\mathbf{S}}_{i}$ and $\widehat{\mathbf{S}}_{j}$ interact thorough the dipole-dipole interaction, described by the following Hamiltonian:

$\hat{H}_{\mathrm{SS}, i j}=\frac{\mu_{0}}{4 \pi} g_{\mathrm{e}}^{2} \mu_{\mathrm{B}}^{2}\left(\frac{\widehat{\mathbf{S}}_{i} \widehat{\mathbf{S}}_{j}}{r^{3}}-\frac{3\left(\widehat{\mathbf{S}}_{i} \mathbf{r}\right)\left(\widehat{\mathbf{S}}_{j} \mathbf{r}\right)}{r^{5}}\right)$,

where $\mathbf{r}$ is the vector between the two electron spins, $r=|\mathbf{r}|$, and $\mu_{0}$ is the vacuum permeability. Note that, for simplicity the $g$ tensor of the electron spin is replaced with the $g_{\mathrm{e}} g$-factor of the free electron. ${ }^{116}$ The spin-spin contribution to the zero-fieldsplitting spin Hamiltonian can be obtained by introducing the total spin operator vector, $\widehat{\mathbf{S}}=\sum_{i} \widehat{\mathbf{S}}_{i}$, and integrating over the spatial degrees of freedom. Note that this is possible when the 
spatial and spin wavefunctions are separable, i.e. no spin-orbit interaction mixes these two degrees of freedom. The spin Hamiltonian can be written as

$\hat{H}_{\text {SS,ZFS }}=\widehat{\mathbf{S} D \widehat{\mathbf{S}}}$,

where $\mathbf{D}$ is the $3 \times 3$ zero-field-splitting tensor. Components of the zero-field-splitting tensor can be calculate from the two particle spin density matrix $\rho_{2}\left(\mathbf{r}_{1}, \mathbf{r}_{2}\right)$ as

$D_{a b}=\frac{1}{2} \frac{\mu_{0}}{4 \pi} g_{\mathrm{e}}^{2} \mu_{\mathrm{B}}^{2} \int \rho_{2}\left(\mathbf{r}_{1}, \mathbf{r}_{2}\right) \frac{r^{2} \delta_{a b}-3 r_{a} r_{b}}{r^{5}} \mathrm{~d} \mathbf{r}_{1} \mathrm{~d} \mathbf{r}_{2}$,

where $r_{a}$ and $r_{b}$ are the Cartesian coordinates of $\mathbf{r}=\left|\mathbf{r}_{1}-\mathbf{r}_{2}\right|$. In axial symmetric cases the splitting of the spin states can be parameterized by a single parameter, $D=\frac{3}{2} D_{z z}$, and the ZFS spin Hamiltonian simplifies to

$\hat{H}_{\mathrm{SS}, \mathrm{ZFS}}^{\text {axial sym. }}=D\left(\hat{S}_{z}^{2}-\frac{S(S+1)}{3}\right)$,

where $S$ is the total spin and $S_{z}$ is the eigenvalue of the component along $z$ quantization axis.

The two particle spin density matrix can be obtained in different wave function-based approaches, as it is generally carried out in quantum chemistry calculations for molecules. ${ }^{119}$ However, it is only approximated in point defect calculations with periodic boundary conditions. ${ }^{120}$ In DFT only density-related quantities can be determined in a consistent way. The density matrix can be approximated by using the Slater determinant of the Kohn-Sham eigenstates of the considered system. This approximation is suitable only when the ground state wave-function is accurately represented by a single Slater determinant ${ }^{120,121}$

$$
D_{a b}=\frac{1}{2} \frac{\mu_{0}}{4 \pi} \frac{g_{\mathrm{e}}^{2} \mu_{\mathrm{B}}^{2}}{S(2 S-1)} \sum_{i>j}^{\text {occupied }} X_{i j} \int\left|\Phi_{i j}\left(\mathbf{r}_{1}, \mathbf{r}_{2}\right)\right|^{2 r^{2} \delta_{a b}-3 r_{a} r_{b}} \mathrm{dr}_{1} \mathrm{~d} \mathbf{r}_{2},
$$

where $\Phi_{i j}\left(\mathbf{r}_{1}, \mathbf{r}_{2}\right)=\frac{1}{\sqrt{2}}\left(\phi_{i}\left(\mathbf{r}_{1}\right) \phi_{j}\left(\mathbf{r}_{2}\right)-\phi_{j}\left(\mathbf{r}_{1}\right) \phi_{i}\left(\mathbf{r}_{2}\right)\right)$ the Slater determinant of Kohn-Sham states $i$ and $j$ and $X_{i j}$ is either 1 or -1 for KS states of the same or different spin channels, respectively. Note that in DFT the Kohn-Sham states are not spin restricted, i.e. the states in the two spin channels are independent from each other. Consequently, not only the unpaired Kohn-Sham states but also the rest of the occupied states can contribute to the spin density and the ZFS. ${ }^{120,121}$ In order to account for these effects, the summation in Eq. (11) includes all pairs of the occupied states.

To the best of our knowledge, the first implementation and point defect calculations were carried out by Rayson et al. ${ }^{120,121}$. In the latter publication an efficient implementation was presented for the plane wave basis set, which was later utilized in other publications too. ${ }^{122,123}$ In all of these early implementations pseudopotentials and pseudowavefunctions were used. The theory of Rayson et al. was recently extended to the PAW method to include corrections from the core region. ${ }^{124,125}$

We would like to draw attention to the fact that the dipole-dipole interaction is long ranged, i.e. it goes with $1 / r^{3}$, thus the finite size effect can be present in periodic supercells. Biktagirov et al. ${ }^{125}$ observed notable effect for point defects in diamond and cubic 3C-SiC. Note that ZFS is a tensor quantity, thus the interaction with the periodic images depends not only on the distances but on the arrangement of the replicas, or equally, on the shape and symmetry of the supercell. To investigate these important aspects that influence the numerical accuracy and to propose possible correction schemes, additional studies are required.

Recently, the ground state ZFS tensor calculations were successfully applied in point defect configuration identification studies, $^{53,65,115}$ where $1-20 \%$ error were observed when the
Table 4. First principles and experimental ground state ZFS values $D$ of selected point defects that are used or proposed as qubits in semiconductors

\begin{tabular}{|c|c|c|c|c|c|}
\hline Host & defect & $D^{\mathrm{ONCV}}$ & $D^{\mathrm{PAW}-\mathrm{ps}}$ & $D^{\mathrm{PAW}}$ & $D^{\exp }$ \\
\hline Diamond & $\mathrm{NV}^{-}$ & $3.03^{123}$ & $2.90^{123}, 2.854^{122}$ & $3.08^{125}$ & $2.88^{63}$ \\
\hline $4 \mathrm{H}-\mathrm{SiC}$ & hh divacancy & $1.682^{123}$ & $\begin{array}{l}1.387^{123} \\
1.358^{127^{\prime}}\end{array}$ & - & $1.336^{49}$ \\
\hline $4 \mathrm{H}-\mathrm{SiC}$ & $\begin{array}{l}\mathrm{V}_{\mathrm{Si}}(-) \text { at } k- \\
\text { site }\end{array}$ & - & $0.0333^{65}$ & - & $0.035^{156}$ \\
\hline 3C-SiC & $\mathrm{N}_{\mathrm{C}} \mathrm{V}_{\mathrm{Si}}(-)$ & - & $1.409^{115}$ & $1.74^{125}$ & $1.303^{115}$ \\
\hline
\end{tabular}

values are compared with experimental results (see Table 4). In all of these applications PBE exchange-correlation functional was used. Note that the results obtained from pseudowavefunctions without PAW contributions compare best with the experimental values. This surprisingly good results must be a consequence of error cancellations in these calculations. ${ }^{123}$ Note also that the current implementations may not be suitable for defects with S2, where higher order terms are expected (see Table 3), and when the $g$-tensor deviates considerably from $g_{e} \mathcal{I}$ of the free electron. $^{116}$

ZFS of point defect qubits is a key quantity to measure variations of the external degrees of freedom. Recently, pressure, $^{122,123}$ strain, $^{126}$ electric field, ${ }^{127}$ and temperature dependence $^{122}$ of the ZFS were successfully studied by first principles calculations.

So far less attention has been paid to the excited state ZFS calculations, where additional considerations are required. As we mentioned in Section 2, ZFS tensor can be obtained only when the spin and spatial degrees of freedom are separable, i.e. spin-orbit interaction is negligible, and the considered states can be described by a single Slater determinant. None of these conditions are satisfied in the low-temperature fine structure of the ${ }^{3} E$ optically excited state of the NV center ${ }^{60,62}$ and divacancy, ${ }^{58}$ thus spin-spin coupling alone cannot describe the low temperate fine structure. On the other hand, according to the accepted theory of the ${ }^{3} E$ states of these defects, at high temperature the spin-orbit interaction and non-axial component of the spin-spin interaction averages out due to the atomic motion of the dynamic Jahn-Teller excited state configuration, ${ }^{62}$ and thus one can obtain states that are good eigenstates of $\hat{S}_{z}$ and $\hat{S}_{z}^{2}$. The ZFS of the NV center and divacancy qubits can be approximated by Kohn-Sham orbitals in constrained occupation DFT and subsequent symmetrization of the $\mathbf{D}$ matrix to mimic the motion averaging and obtain effective $C_{3 v}$ symmetry.

In Table 5, we collected the results of excited state ZFS calculations using PAW pseudowavefunctions obtained by PBE exchange correlation functional. ${ }^{128}$. Except for the NV center in diamond, the theoretical values substantially overestimate the experimental ones. A possible source of this discrepancy can be the neglect of electron-phonon coupling and the vibrational part of the wavefunction. These results as well as the enormous temperature dependence of the excited state ZFS of silicon vacancy ${ }^{66}$ evidence the need for extending the theory of excited state ZFS calculations.

\section{Spin-orbit coupling parameters}

Spin-orbit interaction, due to the relativistic coupling of electron angular motion and the electron spin, has already appeared in 
Table 5. First principles and experimental excited state ZFS values $D$ of selected point defects qubits

\begin{tabular}{llll}
\hline Host & Defect & $D^{\text {PAW-ps }}$ & $D^{\text {exp. }}$ \\
\hline Diamond & $\mathrm{NV}(-)$ & $1.71^{128}$ & $1.43^{157}$ \\
$4 \mathrm{H}-\mathrm{SiC}$ & $h h$ divacancy & $1.33^{128}$ & $0.84^{27}$ \\
$4 \mathrm{H}-\mathrm{SiC}$ & $\mathrm{V}_{\mathrm{Si}}(-)$ at the $k$-site & $2.19^{128}$ & $0.215-0.503^{66}$ \\
\hline
\end{tabular}

PAW-ps denotes calculations of PAW potential with only pseudowavefunction contributions to the ZFS. All values are given in $\mathrm{GHz}$ unit

previous discussion of the $g$-tensor and zero-field-splitting. In this section, we discuss first principles calculation of spin-orbit coupling parameters of point defect qubits in semiconductors that can be used to analyze the fine structure of the states and to investigate possible spin-selective transitions between different defect states.

The spin-orbit interaction Hamiltonian in zero-order approximation can be written as ${ }^{60}$

$\hat{H}_{\mathrm{SO}}=\frac{1}{2} \frac{1}{c^{2} m_{\mathrm{e}}^{2}} \sum_{i}\left(\nabla_{i} V \times \widehat{\mathbf{p}}_{i}\right) \widehat{\mathbf{S}}_{i}$,

where $V$ is the nuclear potential energy, $m_{\mathrm{e}}$ is the electron mass, and $\widehat{\mathbf{p}}_{i}$ and $\widehat{\mathbf{S}}_{i}$ are the momentum and spin of electron $i$. The elements of the orbital operator vector $\widehat{\mathbf{O}}=\nabla_{i} V \times \widehat{\mathbf{p}}_{i}$ can be calculated from the Kohn-Sham orbitals. Note that the crystal field of a solid breaks the spherical symmetry of the spin-orbit interaction. In low symmetry case the interaction Hamiltonian can be rewritten as ${ }^{60}$

$\hat{H}_{\mathrm{SO}}=\sum_{i} \lambda_{x} \hat{L}_{i, x} \hat{S}_{i, x}+\lambda_{y} \hat{L}_{i, y} \hat{S}_{i, y}+\lambda_{z} \hat{L}_{i, z} \hat{S}_{i, z}$

where $\lambda_{a}$ for $a \in\{x, y, z\}$ are the spin-orbit interaction parameters. In $C_{3 v}$ symmetry $\lambda_{\perp}=\lambda_{x}=\lambda_{y}$ and $\lambda_{\|}=\lambda_{z}$ are the basal or non-axial and axial parameters of the interaction, respectively. The former parameter is dominantly responsible for the mixing of different spin states, e.g. triplet and singlet states, while axial parameter is mainly responsible for the splitting and shift of different spin-orbit coupled states. Note that the most frequently used $\lambda \widehat{\mathbf{L S}}$ form is only recovered in higher symmetry cases when $\lambda=\lambda_{\perp}=\lambda_{\|}$. Note also that the above formula inherently includes the assumption that $\nabla_{i} V$ is identical for all the electronic states. This may be violated when systems of different atomic species are considered. In standard implementations available in first principles codes no such approximation is taken, however, the anisotropy of the spin-orbit interaction is generally neglected. ${ }^{129-131}$

In light element hosts, such as diamond and $\mathrm{SiC}$, the spin-orbit coupling energy can be very small for localized point defect states, generally in the order of $10-100 \mu \mathrm{eV}(\approx \mathrm{GHz})$. Furthermore, axial spin-orbit interaction is non-zero for states of non-zero effective spin and orbital momentum. Most often, the ground state of point defect qubits have no effective angular momentum, thus no axial spin-orbit contribution can be observed in the ground state ZFS. ${ }^{50,60,62,67}$

Spin-orbit interaction calculations for the NV center and for other potential qubits, group-IV-vacancy color centers in diamond, have been pioneered by Thiering et al. ${ }^{132,133}$ The axial spin-orbit interaction strength in the excited state can be obtained both from total energy difference calculations and from the splitting of Kohn-Sham states in constrained occupation non-collinear DFT calculations. $^{132}$ To accurately determine small values of the axial spin-orbit interaction, the calculations require high numerical convergence and accuracy. Finite size effect turned to be crucial for spin-orbit interaction calculations ${ }^{132}$ (see Fig. 4). Thiering et al. 132 attributed the observed finite size effect to the overlap of the defect states and used an exponential fit to eliminate supercell

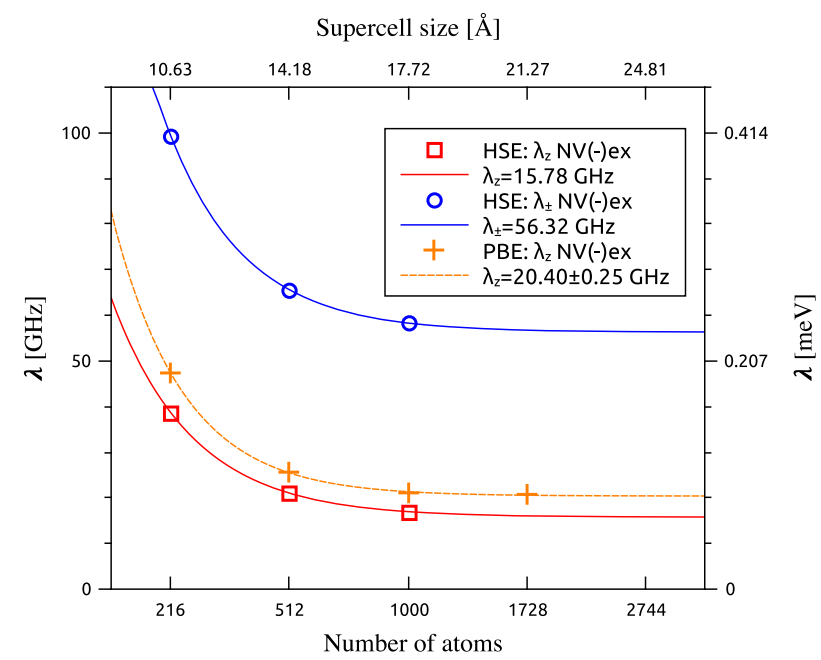

Fig. 4 Convergence of the axial spin-orbit coupling parameter $\lambda$ in the excited state of NV center. Horizontal axes shows the supercell size and the number of carbon atoms in the defect-free supercells. The figure is reprinted with permission from ref. ${ }^{132}$ Copyright (2017) by the American Physical Society

size dependence of the $\lambda_{z}$. Note that axial spin-orbit coupling parameter calculations are only possible in the $\Gamma$-point of the Brillouin zone, as the dispersion and splitting of the defect states in low symmetry $k$-points may be larger than the axial spin-orbit splitting.

It is important to mention that due to the dynamic Jahn-Teller nature of the excited states of the considered defects, the calculated axial spin-orbit parameter cannot be directly compared with the experimental values. ${ }^{132}$ The efficient electron-phonon coupling reduces the angular momentum of the electrons in the excited state. By taking into account this damping effect, the calculated axial spin-orbit parameters compare well with the experimental values. ${ }^{132,133}$ On the other hand, the calculation of the non-axial component of the spin-orbit interaction requires further investigation and development. ${ }^{132}$

Hyperfine tensor calculation

The hyperfine interaction tensor describes the coupling of nuclear spin to the electron spin density of the point defect. As spin density is generally a unique feature of paramagnetic point defects, the hyperfine structure is an important fingerprint that can be utilized in point defect configuration identification. Indeed hyperfine interaction is probably the most frequently calculated spin-dependent quantity of point defects in semiconductors.

Considering only linear term in spin operators, the hyperfine spin Hamiltonian of a single electron spin-nuclear spin pair can be written as

$H_{\text {hyp }}=\widehat{\mathbf{S}} \widehat{A \mathbf{I}}$,

where $\mathbf{A}$ is the hyperfine tensor and $\widehat{\mathbf{I}}$ is the nuclear spin vector operator. When the electron spin density is non-zero at the place of the considered nuclear spin, the hyperfine tensor elements are defined by the sum of two terms

$$
\begin{gathered}
A_{a b}=\frac{2 \mu_{0}}{3} g_{\mathrm{e}} \mu_{\mathrm{B}} g_{\mathrm{N}} \mu_{\mathrm{N}} \frac{\sigma(\mathbf{R})}{S} \\
+\frac{\mu_{0}}{4 \pi} g_{\mathrm{e}} \mu_{\mathrm{B}} g_{\mathrm{N}} \mu_{\mathrm{N}} \frac{1}{S} \int \frac{3 r_{a} r_{b}-r^{2} \delta_{a b}}{r^{5}} \sigma(\mathbf{r}) d \mathbf{r},
\end{gathered}
$$

where $\sigma(\mathbf{r})$ is the electron spin density, $\mathbf{r}$ is the vector between the electron spin and nuclear spin at $\mathbf{R}, g_{\mathrm{N}}$ is the nuclear $g$-factor, and $\mu_{N}$ is the nuclear magneton. The first term on the right-hand side of Eq. (15) is the Fermi contact term that describes isotropic magnetic interaction between the spins, while the second term on 
the right-hand side of Eq. (15) is the anisotropic, long-ranged dipole-dipole interaction term. Note that in Eq. (15), we approximated the electron spin $g$-tensor with $g_{\mathrm{e}}{ }^{116}$

In case of an axial symmetric hyperfine interaction, i.e. when the nuclear spin is located on the symmetry axis of the systems, which becomes the quantization axis as well, the hyperfine tensor is diagonal with diagonal element $A_{x x}=A_{y y}=A_{\perp}$ and $A_{z z}=A_{\| l}$. These parameters can be expressed by the Fermi-contact term $a$ and a simplified dipolar coupling term $b$ as $A_{\perp}=a-b$ and $A_{\|}=a+2 b$.

In the first implementation for DFT calculations with periodic boundary conditions by Van de Walle et al. ${ }^{134,135}$, pseudopotentials were used and only the axial symmetric hyperfine interaction parameters were calculated. This approach was successfully applied in the identification of electron paramagnetic resonance (EPR) centers in semiconductors, see for example the citations of ref. ${ }^{135}$ Later, the theory was extended to Greens' function ${ }^{136}$ calculations and PAW full hyperfine tensor calculations. ${ }^{79,137}$ According to recent numerical tests, HSE06 hybrid functional hyperfine calculations including core polarization correction $^{79,138,139}$ provides the most accurate results. $^{79}$

Hyperfine interaction calculations have been carried out for the most important point defect qubits, such as the NV center, ${ }^{79,140}$ divacancy, ${ }^{27}$ and silicon vacancy. ${ }^{65,79}$ Recently, hyperfine tensors were calculated not only for the first and second neighbor nuclei sites, but also for more distant nuclei sites that are usually not resolvable in experiments. ${ }^{65,141}$

Similarly to the case of electron spin-electron spin dipolar interaction, finite size effects are expected in the hyperfine values that are calculated with periodic boundary conditions. Indeed, using the implementation provided in ref. ${ }^{79}$, considerable finite size effects where reported in ref. ${ }^{53}$ (see Fig. 5). As can be seen, the relative error increases for nuclei sites located farther away from a divacancy point defect qubit. Importantly, both the Fermi contact and the dipole-dipole interaction terms exhibit finite size effects, which however rapidly reduce with increasing supercell size. ${ }^{53}$ These observations suggest that the overlap of the defects states is responsible for the reported finite size effects. Consequently, accurate calculation of hyperfine tensor of distant nuclei sites is only possible in large supercells, where the overlap of the defect states is negligible.

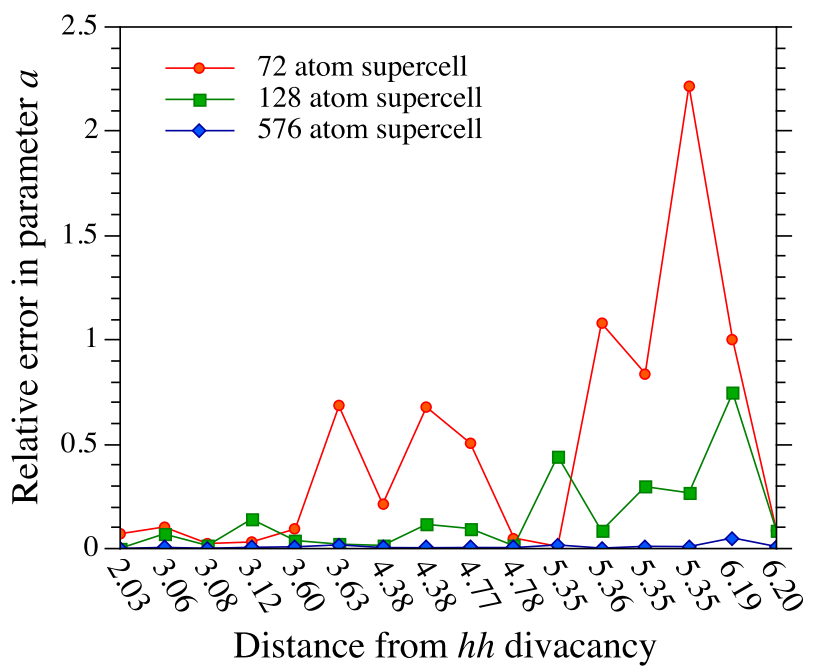

Fig. 5 Relative error of the calculated Fermi contact hyperfine interaction strength for various nuclei sites around a $h h$ divacancy in $4 \mathrm{H} \mathrm{SiC}$ (data from ref. ${ }^{53}$ ). On the horizontal axis the distances from the silicon vacancy site of the divacancy are given. Calculations are carried out in 72, 128, and 576 atom supercell using PBE functional. Deviations in the hyperfine values are measured from the parameters obtained in the absolutely convergent 2400 atom supercell
As a final remark on hyperfine tensor calculations in periodic codes, we mention that finite size effects caused by the longranged dipole-dipole interaction term could be eliminated to a large extent by employing real space integration in Eq. (15). In practice, momentum space integration is commonly utilized, since a six-dimensional real-space integral can be simplified to a threedimensional integral after Fourier transformation. In the case of hyperfine tensor calculations, the real space integral is only threedimensional due to the point-like localization of the nuclei. Consequently, efficient calculations can be carried out on a realspace grid to substantially reduce the artificial effect from the periodic images.

The simulations of shallow donor qubits in silicon quantum dots are especially challenging ${ }^{142}$ due to the delocalization of the defect states and the substantial finite size effects. To overcome some of these problems and, at the same time, to investigate the quantum confinement effects, cluster models are frequently used in hyperfine parameter calculations of these qubits. ${ }^{43,144}$

\section{Other coupling parameters}

In the previous sections, we discussed first principles calculations of the most important spin-related quantities of point defect qubits. At the same time, there are numerous further coupling terms and coupling parameters that can be derived from relativistic perturbation theory. ${ }^{145}$ Restricting ourselves to terms of $\mathcal{O}\left(a^{0}\right)$ and $\mathcal{O}\left(a^{2}\right)$, where $a \approx 1 / 137$ is the fine-structure constant, the following spin and orbital momentum-related interaction terms can appear: (i) nuclear quadrupole interaction $\left(\mathcal{O}\left(a^{0}\right)\right.$ ), (ii) electron orbital-orbital dipole interaction, (iii) spin-spin contact interaction, (iv) orbital hyperfine interaction, and (v) magnetic field-dependent corrections terms to the spin-orbit, spin-otherorbit, nuclear Zeeman, and orbital-hyperfine interactions. There are available implementations for the nuclear quadruple ${ }^{146,147}$ and orbital hyperfine interaction terms. ${ }^{148-150}$ However, to the best of our knowledge these terms have not been considered in the context of first principles point defect qubit calculations. Investigation of higher-order terms may be important for highly accurate first principles calculations and for studying exotic spin state couplings.

\section{Role of electron-phonon coupling}

Although, electron-phonon coupling is not a spin-dependent phenomena, it still can indirectly affect the expectation values of spin-dependent observables, as we have already seen in the case of spin-orbit interaction in Section 4. In general, when the adiabatic approximation is violated, mixed electronic, and vibronic wavefunctions are required to accurately calculate orbitaldependent spin coupling parameters such as $g$-tensor, zerofield-splitting, and spin-orbit interaction parameters. Therefore, here we shortly review relevant works on the description of electron-phonon coupling in point defect qubits.

Ground or excited states of point defects often exhibit different Jahn-Teller instabilities. In the dynamic Jahn-Teller effect, when the zero-point motion or thermally occupied phonon modes have sufficient energy to continuously drive the system between different Jahn-Teller distorted states, the electron states and phonon modes strongly couple. Recently, model Hamiltonian approach was applied to investigate the triplet excited state of the NV center in diamond, which is a dynamic Jahn-Teller system, where the partially occupied $e$ single particle state couples to a localized $E$ vibrational mode. Abtew et al. ${ }^{151}$ and Thiering et al. ${ }^{132}$ applied a so-called $e \otimes E$ model, in which a model potential energy surface of the $e$ electronic states over generalized coordinate space of the $E$ vibrational mode is parameterized by constrained occupation DFT calculations. The electron-phonon coupling potential established this way is added to the Hamiltonian of the $e \otimes E$ model system and solved by exact 
diagonalization. Even in the vibrational ground state, different electronic states of different angular momentum are mixed with vibronic states. As a consequence, the $L=2$ angular momentum of the excited state electronic configuration is quenched down by $70 \%$ in the vibronic ground state, resulting in theoretical axial spin-orbit coupling parameter compares well with the experiment. ${ }^{132}$ Note that similar theory should be applied to the $g$ tensor calculation in the excited state. The zero-field-splitting of the vibronic ground state has not been investigated yet.

\section{SUMMARY AND OUTLOOK}

Point defect quantum bits in semiconductors are among the most recent inventions of solid-state physicists and material scientists that may serve as building units for room temperature controlled quantum systems in semiconductors. Additionally, point defect quantum bits provide an interface for investigating materials and molecules with nanoscale spatial resolution. As these elementary solid state quantum devices can be directly and quantitatively studied by first principles calculations, theoretical studies hold a great promise for fast development of these areas.

As we have shown here, methodology and implementations for the calculations of the most important ground state spindependent quantities have already been demonstrated in the literature. In general, applications of these methods show considerable accuracy and predictive power, which is encouraging for broadening the range of simulations. On the other hand, there is a great need in further developments. In some cases, the limitations of the currently used method or technical requirements of completely convergent calculations are still unknown. Furthermore, higher-order coupling term calculations might be considered in the future.

Calculations of excited state spin-dependent properties, however, still remain quite challenging. The reason to this is twofold. First, proper excited state electronic structure calculations require to go beyond conventional applications of the DFT. Second, the electron-phonon coupling tends to play an important role in some properties of the excited states. Calculation of the spindependent quantities will become significantly more reliable upon overcoming the above-mentioned methodological challenges.

\section{Future applications}

Finally, we would like to call attention to two possible future applications for the existing and required first principles methods mentioned in this Review Article.

First principles predictions of point defect qubits. There are numerous two-dimensional and three-dimensional semiconducting materials that have been synthesized so far and their number is increasing day by day. Many of these materials can host paramagnetic point defects that may exhibit potential for implementing novel point defect qubits. Using first principles calculations is the most suitable approach for fast investigation of this vast unexplored field. Requirements for systematic point defect search have been discussed in the literature. ${ }^{53}$ Moreover, it has been shown that computationally efficient methods can be used for high-throughput screening of point defect by calculating the defects spin states and basic optical properties. ${ }^{53,152}$ The evaluation of candidate paramagnetic point defects real potential for qubit applications, however, requires more detailed studies by using methods summarized in this paper. For such systematic point defect qubit search, appropriate highly automated search algorithms are yet to be developed.

Ab initio support for predictive spin dynamic simulations. Simulations in the framework of model spin Hamiltonian for qualitative understanding of basic functionalities of point defect qubits is an other emerging direction for theoretical studies. These simulations generally rely on spin coupling and other system-specific parameters that at present are usually obtained from experimental measurements. Accurate measurement of all of the required parameters is cumbersome and not always possible, thus spin Hamiltonian models frequently contain free parameters as well. Consequently, quantitative predictive power and transferability of these methods are highly limited.

On the other hand, first principles calculations of spin-related parameters of point defect qubits may take over the role of experimental measurements. Combining ab initio calculations with model spin Hamiltonian approaches may results in novel methodologies that allow for parameter free quantitatively predictive spin dynamic simulations. Such method would be highly important not only for quantitative understanding of the physics of point defect spins but also for modeling the operation and applications of point defect qubit candidates.

Indeed, in recent spin dynamic simulations for optical dynamic nuclear polarization (ODNP) thorough divacancy quantum bits, ab initio hyperfine tensors were used in the simulations that allowed the prediction of fine structures in the magnetic field dependence of the nuclear spin polarization, which were confirmed in experiment. $^{153}$ This example demonstrates the potential of ab intio theory-supported spin Hamiltonian approaches.

\section{ACKNOWLEDGEMENTS}

Discussions with Gergő Thiering and Zoltán Bodrog are highly appreciated. Support from the Swedish Government Strategic Research Areas in Materials Science on Functional Materials at Linköping University (Faculty Grant SFO-Mat-LiU No. 200900971) and Knut \& Alice Wallenberg Foundation New States of Matter 2014-2019 (COTXS) is gratefully acknowledged. Analysis of first-principles calculations of defect properties was supported by the Ministry of Education and Science of the Russian Federation (Grant no. 14.Y26.31.0005). Applications of the model Hamiltonians were supported by the Ministry of Education and Science of the Russian Federation in the framework of Increase Competitiveness Program of NUST "MISIS" (No. K2-2017-080) implemented by a governmental decree dated 16 March 2013, no. 211. A.G. acknowledges the support from the National Research Development and Innovation Office of Hungary (NKFIH) within the Quantum Technology National Excellence Program (Project no. 2017-1.2.1-NKP-2017-00001), the EU QuantERA projects QMagine and Nanospin (NKFIH Grant Nos. 127889 and 127902, respectively), and the EU H2020 ASTERIQS project.

\section{AUTHOR CONTRIBUTIONS}

The manuscript was written through contributions of all authors. V.I. wrote the paper and I.A.A. and A.G. examined and improved it.

\section{ADDITIONAL INFORMATION}

Competing interests: The authors declare no competing interests.

Publisher's note: Springer Nature remains neutral with regard to jurisdictional claims in published maps and institutional affiliations.

\section{REFERENCES}

1. Ladd, T. D. et al. Quantum computers. Nature 464, 45-53 (2010).

2. Wrachtrup, J. \& Jelezko, F. Processing quantum information in diamond. J. Phys. Condens. Matter 18, S807-S824 (2006).

3. Weber, J. R. et al. Quantum computing with defects. PNAS 107, 8513-8518 (2010).

4. Dutt, M. V. G. et al. Quantum register based on individual electronic and nuclear spin qubits in diamond. Science 316, 1312-1316 (2007).

5. Kane, B. E. A silicon-based nuclear spin quantum computer. Nature 393, 133-137 (1998).

6. Vrijen, R. et al. Electron-spin-resonance transistors for quantum computing in silicon-germanium heterostructures. Phys. Rev. A 62, 012306 (2000).

7. Awschalom, D. D., Bassett, L. C., Dzurak, A. S., Hu, E. L. \& Petta, J. R. Quantum spintronics: engineering and manipulating atom-like spins in semiconductors. Science 339, 1174-1179 (2013). 
8. Tyryshkin, A. M. et al. Electron spin coherence exceeding seconds in high-purity silicon. Nat. Mater. 11, 143-147 (2011).

9. Balasubramanian, $\mathrm{G}$. et al. Ultralong spin coherence time in isotopically engineered diamond. Nat. Mater. 8, 383-387 (2009).

10. Koehl, W. F., Buckley, B. B., Heremans, F. J., Calusine, G. \& Awschalom, D. D. Room temperature coherent control of defect spin qubits in silicon carbide. Nature 479, 84-87 (2011).

11. Widmann, M. et al. Coherent control of single spins in silicon carbide at room temperature. Nat. Mater. 14, 164-168 (2015).

12. Kucsko, G. et al. Nanometre-scale thermometry in a living cell. Nature $\mathbf{5 0 0}$ 54-58 (2013)

13. Iwasaki, T. et al. Direct nanoscale sensing of the internal electric field in operating semiconductor devices using single electron spins. ACS Nano 11 1238-1245 (2017).

14. Barson, M. S. J. et al. Nanomechanical sensing using spins in diamond. Nano Lett. 17, 1496-1503 (2017).

15. Balasubramanian, G. et al. Nanoscale imaging magnetometry with diamond spins under ambient conditions. Nature 455, 648-651 (2008).

16. Mamin, H. J. et al. Nanoscale nuclear magnetic resonance with a nitrogenvacancy spin sensor. Science 339, 557-560 (2013).

17. Aslam, N. et al. Nanoscale nuclear magnetic resonance with chemical resolution. Science 357, 67-71 (2017).

18. Wolf, T. et al. Subpicotesla diamond magnetometry. Phys. Rev. X 5, 041001 (2015).

19. Lee, S.-Y., Niethammer, M. \& Wrachtrup, J. Vector magnetometry based on electronic spins. Phys. Rev. B 92, 115201 (2015).

20. Ledbetter, M., Jensen, K., Fischer, R., Jarmola, A. \& Budker, D. Gyroscopes based on nitrogen-vacancy centers in diamond. Phys. Rev. A 86, 052116 (2012).

21. Ajoy, A. \& Cappellaro, P. Stable three-axis nuclear-spin gyroscope in diamond. Phys. Rev. A 86, 062104 (2012).

22. Childress, L., Taylor, J. M., Sørensen, A. S. \& Lukin, M. D. Fault-tolerant quantum communication based on solid-state photon emitters. Phys. Rev. Lett. 96, 070504 (2006).

23. Bernien, $\mathrm{H}$. et al. Heralded entanglement between solid-state qubits separated by three metres. Nature 497, 86-90 (2013).

24. Hensen, B. et al. Loophole-free bell inequality violation using electron spins separated by 1.3 kilometres. Nature 526, 682-686 (2015).

25. Tosi, G. et al. Silicon quantum processor with robust long-distance qubit couplings. Nat. Commun. 8, 450 (2017).

26. Jacques, V. et al. Dynamic polarization of single nuclear spins by optical pumping of nitrogen-vacancy color centers in diamond at room temperature. Phys. Rev. Lett. 102, 057403 (2009).

27. Falk, A. L. et al. Optical polarization of nuclear spins in silicon carbide. Phys. Rev. Lett. 114, 247603 (2015)

28. King, J. P. et al. Room-temperature in situ nuclear spin hyperpolarization from optically pumped nitrogen vacancy centres in diamond. Nat. Commun. 6, 8965 (2015).

29. Tyryshkin, A. M., Lyon, S. A., Astashkin, A. V. \& Raitsimring, A. M. Electron spin relaxation times of phosphorus donors in silicon. Phys. Rev. B 68, 193207 (2003).

30. Mohammady, M. H., Morley, G. W. \& Monteiro, T. S. Bismuth qubits in silicon: The role of EPR cancellation resonances. Phys. Rev. Lett. 105, 067602 (2010).

31. Klimov, P. V., Falk, A. L., Christle, D. J., Dobrovitski, V. V. \& Awschalom, D. D. Quantum entanglement at ambient conditions in a macroscopic solid-state spin ensemble. Sci. Adv. 1, e1501015 (2015).

32. Aharonovich, I. et al. Two-level ultrabright single photon emission from diamond nanocrystals. Nano. Lett. 9, 3191-3195 (2009).

33. Castelletto, S. et al. A silicon carbide room-temperature single-photon source. Nat. Mater. 13, 151-156 (2014).

34. Kolesov, R. et al. Optical detection of a single rare-earth ion in a crystal. Nat. Commun. 3, 1029 (2012).

35. Maze, J. R. et al. Nanoscale magnetic sensing with an individual electronic spin in diamond. Nature 455, 644-647 (2008).

36. Buckley, B. B., Fuchs, G. D., Bassett, L. C. \& Awschalom, D. D. Spin-light coherence for single-spin measurement and control in diamond. Science 330, 1212-1215 (2010).

37. Robledo, L. et al. High-fidelity projective read-out of a solid-state spin quantum register. Nature 477, 574-578 (2011).

38. Christle, D. J. et al. Isolated electron spins in silicon carbide with millisecond coherence times. Nat. Mater. 14, 160-163 (2015).

39. Soltamov, V. A., Soltamova, A. A., Baranov, P. G. \& Proskuryakov, I. I. Room temperature coherent spin alignment of silicon vacancies in $4 \mathrm{H}-$ and $6 \mathrm{H}-\mathrm{SiC}$. Phys. Rev. Lett. 108, 226402 (2012).

40. Becker, J. N. \& Becher, C. Coherence properties and quantum control of silicon vacancy color centers in diamond. Phys. Status Solidi A 214, 1700586 (2017).
41. Rose, B. C. et al. Observation of an environmentally insensitive solid-state spin defect in diamond. Science 361, 60-63 (2018).

42. Siyushev, P. et al. Optical and microwave control of germanium-vacancy center spins in diamond. Phys. Rev. B 96, 081201 (2017).

43. Iwasaki, T. et al. Tin-vacancy quantum emitters in diamond. Phys. Rev. Lett. 119 253601 (2017)

44. Morse, K. J. et al. A photonic platform for donor spin qubits in silicon. Sci. Adv. 3, e1700930 (2017).

45. Siyushev, P. et al. Coherent properties of single rare-earth spin qubits. Nat. Commun. 5, 3895 (2014).

46. Fraval, E., Sellars, M. J. \& Longdell, J. J. Dynamic decoherence control of a solidstate nuclear-quadrupole qubit. Phys. Rev. Lett. 95, 030506 (2005).

47. Saeedi, K. et al. Room-temperature quantum bit storage exceeding 39 minutes using ionized donors in silicon-28. Science 342, 830-833 (2013).

48. Awschalom, D. D., Hanson, R., Wrachtrup, J. \& Zhou, B. B. Quantum technologies with optically interfaced solid-state spins. Nat. Photon. 12, 516-527 (2018).

49. Falk, A. L. et al. Polytype control of spin qubits in silicon carbide. Nat. Commun. 4, 1819 (2013).

50. Gali, A., Gällström, A., Son, N. \& Janzén, E. Theory of neutral divacancy in SiC: a defect for spintronics. Mater. Sci. Forum 645-648, 395-397 (2010).

51. Gali, A., Fyta, M. \& Kaxiras, E. Ab initio supercell calculations on nitrogen-vacancy center in diamond: electronic structure and hyperfine tensors. Phys. Rev. B 77, 155206 (2008)

52. Gordon, L., Janotti, A. \& Van de Walle, C. G. Defects as qubits in $3 C$ - and $4 H$ -SiC. Phys. Rev. B 92, 045208 (2015).

53. Davidsson, J. et al. First principles predictions of magneto-optical data for semiconductor point defect identification: the case of divacancy defects in $4 \mathrm{H}$ SiC. New J. Phys. 20, 023035 (2018).

54. Loubser, J. H. N. \& van Wyk, J. A. Electron spin resonance in the study of diamond. Rep. Prog. Phys. 41, 1201 (1978).

55. Son, N. et al. Divacancy in 4H-SiC. Phys. Rev. Lett. 96, 055501 (2006).

56. Tamarat, P. et al. Spin-flip and spin-conserving optical transitions of the nitrogen-vacancy centre in diamond. New J. Phys. 10, 045004 (2008).

57. Batalov, A. et al. Low temperature studies of the excited-state structure of negatively charged nitrogen-vacancy color centers in diamond. Phys. Rev. Lett. 102, 195506 (2009).

58. Christle, D. J. et al. Isolated spin qubits in $\mathrm{SiC}$ with a high-fidelity infrared spin-tophoton interface. Phys. Rev. X 7, 021046 (2017).

59. Rogers, L. J., McMurtrie, R., Sellars, M. \& Manson, N. B. Time-averaging within the excited state of the nitrogen-vacancy centre in diamond. New J. Phys. 11, 063007 (2009)

60. Maze, J. R. et al. Properties of nitrogen-vacancy centers in diamond: the group theoretic approach. New J. Phys. 13, 025025 (2011).

61. Doherty, M. W., Manson, N. B., Delaney, P. \& Hollenberg, L. C. L. The negatively charged nitrogen-vacancy centre in diamond: the electronic solution. New J. Phys. 13, 025019 (2011).

62. Doherty, M. W. et al. The nitrogen-vacancy colour centre in diamond. Phys. Rep. 528, 1-45 (2013).

63. Jelezko, F., Gaebel, T., Popa, I., Gruber, A. \& Wrachtrup, J. Observation of coherent oscillations in a single electron spin. Phys. Rev. Lett. 92, 076401 (2004)

64. Gali, A. Excitation spectrum of point defects in semiconductors studied by timedependent density functional theory. J. Mat. Res. 27, 897 (2012).

65. Ivády, V. et al. Identification of Si-vacancy related room-temperature qubits in 4H silicon carbide. Phys. Rev. B 96, 161114 (2017)

66. Anisimov, A. N. et al. Optical thermometry based on level anticrossing in silicon carbide. Sci. Rep. 6, 33301 (2016)

67. Soykal, O. O., Dev, P. \& Economou, S. E. Silicon vacancy center in 4H-SiC: electronic structure and spin-photon interfaces. Phys. Rev. B 93, 081207 (2016).

68. Jelezko, F. et al. Observation of coherent oscillation of a single nuclear spin and realization of a two-qubit conditional quantum gate. Phys. Rev. Lett. 93, 130501 (2004).

69. Hohenberg, P. \& Kohn, W. Inhomogeneous electron gas. Phys. Rev. 136 B864-B871 (1964)

70. Jones, R. O. Density functional theory: Its origins, rise to prominence, and future. Rev. Mod. Phys. 87, 897-923 (2015)

71. Kohn, W. \& Sham, L. J. Self-consistent equations including exchange and correlation effects. Phys. Rev. 140, A1133-A1138 (1965).

72. Kohn, W. Nobel lecture: Electronic structure of matter-wave functions and density functionals. Rev. Mod. Phys. 71, 1253-1266 (1999).

73. Seidl, A., Görling, A., Vogl, P., Majewski, J. A. \& Levy, M. Generalized Kohn-Sham schemes and the band-gap problem. Phys. Rev. B 53, 3764-3774 (1996).

74. Perdew, J. P., Burke, K. \& Ernzerhof, M. Generalized gradient approximation made simple. Phys. Rev. Lett. 77, 3865-3868 (1996).

75. Heyd, J., Scuseria, G. E. \& Ernzerhof, M. Hybrid functionals based on a screened Coulomb potential. J. Chem. Phys. 118, 8207 (2003) 
76. Heyd, J., Scuseria, G. E. \& Ernzerhof, M. Erratum: "hybrid functionals based on a screened coulomb potential" [J. Chem. Phys. 118, 8207 (2003)]. J. Chem. Phys. 124, 219906 (2006)

77. Henderson, T. M., Paier, J. \& Scuseria, G. E. Accurate treatment of solids with the HSE screened hybrid. Phys. Status Solidi B 248, 767-774 (2010).

78. Deák, P., Aradi, B., Frauenheim, T., Janzén, E. \& Gali, A. Accurate defect levels obtained from the HSE06 range-separated hybrid functional. Phys. Rev. B 81, 153203 (2010)

79. Szász, K., Hornos, T., Marsman, M. \& Gali, A. Hyperfine coupling of point defects in semiconductors by hybrid density functional calculations: the role of core spin polarization. Phys. Rev. B 88, 075202 (2013).

80. Perdew, J. P., Parr, R. G., Levy, M. \& Balduz, J. L. Density-functional theory for fractional particle number: derivative discontinuities of the energy. Phys. Rev. Lett. 49, 1691-1694 (1982).

81. Iori, F., Gatti, M. \& Rubio, A. Role of nonlocal exchange in the electronic structure of correlated oxides. Phys. Rev. B 85, 115129 (2012).

82. Deák, P. et al. Choosing the correct hybrid for defect calculations: a case study on intrinsic carrier trapping in $\beta-\mathrm{Ga}_{2} \mathrm{O}_{3}$. Phys. Rev. B 95, 075208 (2017).

83. Perdew, J. P. \& Zunger, A. Self-interaction correction to density-functional approximations for many-electron systems. Phys. Rev. B 23, 5048-5079 (1981).

84. Gerosa, M., Bottani, C. E., Valentin, C. D., Onida, G. \& Pacchioni, G. Accuracy of dielectric-dependent hybrid functionals in the prediction of optoelectronic properties of metal oxide semiconductors: a comprehensive comparison with many-body GW and experiments. J. Phys.: Condens. Matter 30, 044003 (2018)

85. Ivády, V., Abrikosov, I. A., Janzén, E. \& Gali, A. Role of screening in the density functional applied to transition-metal defects in semiconductors. Phys. Rev. B 87, 205201 (2013).

86. Ming, W., Yang, D., Li, T., Zhang, L. \& Du, M.-H. Formation and diffusion of metal impurities in perovskite solar cell material $\mathrm{CH}_{3} \mathrm{NH}_{3} \mathrm{Pbl}_{3}$ : implications on solar cell degradation and choice of electrode. Adv. Sci. 5, 1700662 (2017).

87. Walsh, A., Da Silva, J. L. F. \& Wei, S.-H. Theoretical description of carrier mediated magnetism in cobalt doped ZnO. Phys. Rev. Lett. 100, 256401 (2008)

88. Coulter, J. E., Manousakis, E. \& Gali, A. Limitations of the hybrid functional approach to electronic structure of transition metal oxides. Phys. Rev. B 88, 041107 (2013).

89. Ivády, V. et al. Theoretical unification of hybrid-DFT and DFT $+U$ methods for the treatment of localized orbitals. Phys. Rev. B 90, 035146 (2014).

90. Blöchl, P. E. Projector augmented-wave method. Phys. Rev. B 50, 17953-17979 (1994).

91. Beste, A. \& Taylor, D. E. Convergence of Ground and Excited State Properties of Divacancy Defects in $4 \mathrm{H}$-SiC with Computational Cell Size. US Army Research Laboratory ARL-TR-8313 (2018).

92. Gali, A., Janzén, E., Deák, P., Kresse, G. \& Kaxiras, E. Theory of spin-conserving

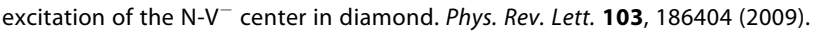

93. Dreyer, C. E., Alkauskas, A., Lyons, J. L., Janotti, A. \& de Walle, C. G. V. Firstprinciples calculations of point defects for quantum technologies. Annu. Rev. Mater. Res. 48, 1-26 (2018).

94. Lany, S. \& Zunger, A. Polaronic hole localization and multiple hole binding of acceptors in oxide wide-gap semiconductors. Phys. Rev. B 80, 085202 (2009).

95. Dabo, I. et al. Koopmans' condition for density-functional theory. Phys. Rev. B 82, 115121 (2010)

96. Perdew, J. P. \& Levy, M. Comment on "significance of the highest occupied kohn-sham eigenvalue". Phys. Rev. B 56, 16021-16028 (1997).

97. Komsa, H.-P., Rantala, T. T. \& Pasquarello, A. Finite-size supercell correction schemes for charged defect calculations. Phys. Rev. B 86, 045112 (2012).

98. Hedin, L. New method for calculating the one-particle Green's function with application to the electron-gas problem. Phys. Rev. 139, A796-A823 (1965).

99. Salpeter, E. E. \& Bethe, H. A. A relativistic equation for bound-state problems. Phys. Rev. 84, 1232-1242 (1951).

100. Ma, Y., Rohlfing, M. \& Gali, A. Excited states of the negatively charged nitrogenvacancy color center in diamond. Phys. Rev. B 81, 041204 (2010).

101. Delaney, P., Greer, J. C. \& Larsson, J. A. Spin-polarization mechanisms of the nitrogen-vacancy center in diamond. Nano Lett. 10, 610-614 (2010).

102. Ranjbar, A. et al. Many-electron states of nitrogen-vacancy centers in diamond and spin density calculations. Phys. Rev. B 84, 165212 (2011).

103. Choi, S., Jain, M. \& Louie, S. G. Mechanism for optical initialization of spin in NV center in diamond. Phys. Rev. B 86, 041202 (2012).

104. Stephens, P. J., Devlin, F. J., Chabalowski, C. F. \& Frisch, M. J. Ab initio calculation of vibrational absorption and circular dichroism spectra using density functional force fields. J. Chem. Phys. 98, 11623-11627 (1994).

105. Bockstedte, M., Schütz, F., Garratt, T., Ivády, V. \& Gali, A. Ab initio description of highly correlated states in defects for realizing quantum bits. npj Quantum Mater. 3, 31 (2018)
106. Aryasetiawan, F. et al. Frequency-dependent local interactions and low-energy effective models from electronic structure calculations. Phys. Rev. B 70, 195104 (2004).

107. Schreckenbach, G. \& Ziegler, T. Calculation of the g-tensor of electron paramagnetic resonance spectroscopy using gauge-including atomic orbitals and density functional theory. J. Phys. Chem. A 101, 3388-3399 (1997).

108. Harriman, J. E. Theoretical Foundations of Electron Spin Resonance (Academic Press, London, 1978).

109. Pickard, C. J. \& Mauri, F. First-principles theory of the EPR $g$ tensor in solids: Defects in quartz. Phys. Rev. Lett. 88, 086403 (2002).

110. Sebastiani, D. \& Parrinello, M. A new ab-initio approach for NMR chemical shifts in periodic systems. J. Phys. Chem. A 105, 1951-1958 (2001).

111. Declerck, R., Van Speybroeck, V. \& Waroquier, M. First-principles calculation of the epr $g$ tensor in extended periodic systems. Phys. Rev. B 73, 115113 (2006).

112. Pickard, C. J. \& Mauri, F. All-electron magnetic response with pseudopotentials: NMR chemical shifts. Phys. Rev. B 63, 245101 (2001)

113. Kadantsev, E. S. \& Ziegler, T. Implementation of a DFT-based method for the calculation of the Zeeman g-tensor in periodic systems with the use of numerical and Slater-type atomic orbitals. J. Phys. Chem. A 113, 1327-1334 (2009).

114. von Bardeleben, H. J., Cantin, J. L., Rauls, E. \& Gerstmann, U. Identification and magneto-optical properties of the NV center in $4 \mathrm{H}-\mathrm{SiC}$. Phys. Rev. B 92, 064104 (2015).

115. von Bardeleben, $\mathrm{H}$. J. et al. NV centers in $3 C, 4 H$, and $6 \mathrm{H}$ silicon carbide: a variable platform for solid-state qubits and nanosensors. Phys. Rev. B 94, 121202 (2016).

116. Weil, J. A. \& Bolton, J. R. Electron Paramagnetic Resonance: Elementary Theory and Practical Applications (John Wiley \& Sons, Hoboken, New Jersey, 2007).

117. McGavin, D. G. \& Tennant, W. C. Higher-order Zeeman and spin terms in the electron paramagnetic resonance spin Hamiltonian; their description in irreducible form using Cartesian, tesseral spherical tensor and Stevens' operator expressions. J. Phys. Condens. Matter 21, 245501 (2009).

118. Simin, D. et al. All-optical dc nanotesla magnetometry using silicon vacancy fine structure in isotopically purified silicon carbide. Phys. Rev. X 6, 031014 (2016).

119. Kaupp, M., Bühl, M. \& Malkin, V. G. Calculation of NMR and EPR Parameters: Theory and Applications (Wiley-VCH, Weinheim, 2004).

120. Rayson, M. J. \& Briddon, P. R. First principles method for the calculation of zerofield splitting tensors in periodic systems. Phys. Rev. B 77, 035119 (2008).

121. Rayson, M., Goss, J. \& Briddon, P. First principles calculation of zero-field splitting tensors. Phys. B 340-342, 673-676 (2003).

122. Ivády, V., Simon, T., Maze, J. R., Abrikosov, I. A. \& Gali, A. Pressure and temperature dependence of the zero-field splitting in the ground state of NV centers in diamond: a first-principles study. Phys. Rev. B 90, 235205 (2014).

123. Seo, H., Ma, H., Govoni, M. \& Galli, G. Designing defect-based qubit candidates in wide-gap binary semiconductors for solid-state quantum technologies. Phys. Rev. Mater. 1, 075002 (2017).

124. Bodrog, Z. \& Gali, A. The spin-spin zero-field splitting tensor in the projectoraugmented-wave method. J. Phys. Condens. Matter 26, 015305 (2014).

125. Biktagirov, T., Schmidt, W. G. \& Gerstmann, U. Calculation of spin-spin zero-field splitting within periodic boundary conditions: towards all-electron accuracy. Phys. Rev. B 97, 115135 (2018).

126. Udvarhelyi, P., Shkolnikov, V. O., Gali, A., Burkard, G. \& Pályi, A. Spin-strain interaction in nitrogen-vacancy centers in diamond. Phys. Rev. B 98, 075201 (2018).

127. Falk, A. L. et al. Electrically and mechanically tunable electron spins in silicon carbide color centers. Phys. Rev. Lett. 112, 187601 (2014).

128. Ivády, V., Abrikosov, I. A. \& Gali, A. unpublished data.

129. Gonze, X. et al. First-principles computation of material properties: the ABINIT software project. Comput. Mater. Sci. 25, 478-492 (2002).

130. Corso, A. D. \& Conte, A. M. Spin-orbit coupling with ultrasoft pseudopotentials: application to Au and Pt. Phys. Rev. B 71, 115106 (2005).

131. Steiner, S., Khmelevskyi, S., Marsmann, M. \& Kresse, G. Calculation of the magnetic anisotropy with projected-augmented-wave methodology and the case study of disordered $\mathrm{Fe}_{1-x} \mathrm{CO}_{x}$ alloys. Phys. Rev. B 93, 224425 (2016).

132. Thiering, G. \& Gali, A. Ab initio calculation of spin-orbit coupling for an NV center in diamond exhibiting dynamic Jahn-Teller effect. Phys. Rev. B 96, 081115 (2017).

133. Thiering, G. \& Gali, A. Ab initio magneto-optical spectrum of group-IV vacancy color centers in diamond. Phys. Rev. X 8, 021063 (2018).

134. Van de Walle, C. G. Structural identification of hydrogen and muonium centers in silicon: First-principles calculations of hyperfine parameters. Phys. Rev. Lett. 64, 669-672 (1990).

135. Van de Walle, C. G. \& Blöchl, P. E. First-principles calculations of hyperfine parameters. Phys. Rev. B 47, 4244-4255 (1993)

136. Gerstmann, U. Ab initio Green's function calculation of hyperfine interactions for shallow defects in semiconductors. Phys. Status Solidi B 248, 1319-1336 (2010). 
137. Blöchl, P. E. First-principles calculations of defects in oxygen-deficient silica exposed to hydrogen. Phys. Rev. B 62, 6158-6179 (2000).

138. Yazyev, O. V., Tavernelli, I., Helm, L. \& Röthlisberger, U. Core spin-polarization correction in pseudopotential-based electronic structure calculations. Phys. Rev. B 71, 115110 (2005).

139. Bahramy, M. S., Sluiter, M. H. F. \& Kawazoe, Y. Pseudopotential hyperfine calculations through perturbative core-level polarization. Phys. Rev. B 76, 035124 (2007).

140. Gali, A. Identification of individual ${ }^{13} \mathrm{C}$ isotopes of nitrogen-vacancy center in diamond by combining the polarization studies of nuclear spins and firstprinciples calculations. Phys. Rev. B 80, 241204 (2009).

141. Ivády, V. et al. Theoretical model of dynamic spin polarization of nuclei coupled to paramagnetic point defects in diamond and silicon carbide. Phys. Rev. B 92 115206 (2015)

142. Smith, J. S. et al. Ab initio calculation of energy levels for phosphorus donors in silicon. Sci. Rep. 7, 6010 (2017)

143. Melnikov, D. V. \& Chelikowsky, J. R. Quantum confinement in phosphorus-doped silicon nanocrystals. Phys. Rev. Lett. 92, 046802 (2004).

144. Yan, B., Rurali, R. \& Gali, Á. Ab initio study of phosphorus donors acting as quantum bits in silicon nanowires. Nano. Lett. 12, 3460-3465 (2012).

145. Manninen, P. Breit-Pauli Hamiltonian and Molecular Magnetic Resonance Properties. Ph.D. thesis, University of Oulu (2004).

146. Petrilli, H. M., Blöchl, P. E., Blaha, P. \& Schwarz, K. Electric-field-gradient calculations using the projector augmented wave method. Phys. Rev. B 57, 14690-14697 (1998).

147. Charpentier, T. The PAW/GIPAW approach for computing NMR parameters: a new dimension added to NMR study of solids. Solid State Nucl. Mag. 40, 1-20 (2011).

148. Blügel, S., Akai, H., Zeller, R. \& Dederichs, P. H. Hyperfine fields of $3 d$ and $4 d$ impurities in nickel. Phys. Rev. B 35, 3271-3283 (1987).

149. Chlan, V. et al. Hyperfine interactions in lutetium iron garnet. J. Appl. Phys. 99 $08 \mathrm{M} 903$ (2006).

150. Chlan, V., Štěpánková, H., Řezníček, R. \& Novák, P. Anisotropy of hyperfine interactions as a tool for interpretation of NMR spectra in magnetic materials. Solid State Nucl. Mag. 40, 27-30 (2011).
151. Abtew, T. A. et al. Dynamic Jahn-Teller effect in the $\mathrm{NV}^{-}$center in diamond. Phys. Rev. Lett. 107, 146403 (2011).

152. Lucatto, B., Assali, L. V. C., Pela, R. R., Marques, M. \& Teles, L. K. General procedure for the calculation of accurate defect excitation energies from DFT-1/2 band structures: the case of the $\mathrm{NV}^{-}$center in diamond. Phys. Rev. B 96, 075145 (2017).

153. Ivády, V. et al. High-fidelity bidirectional nuclear qubit initialization in SiC. Phys. Rev. Lett. 117, 220503 (2016).

154. Davies, G. \& Hamer, M. F. Optical studies of the $1.945 \mathrm{eV}$ vibronic band in diamond. Proc. R. Soc. Lond. Ser. A 348, 285 (1976).

155. Rogers, L. J., Armstrong, S., Sellars, M. J. \& Manson, N. B. Infrared emission of the NV centre in diamond: Zeeman and uniaxial stress studies. New J. Phys. 10, 103024 (2008).

156. Wagner, M. et al. Electronic structure of the neutral silicon vacancy in $4 \mathrm{H}$ and $6 \mathrm{H}$ SiC. Phys. Rev. B 62, 16555-16560 (2000).

157. Fuchs, G. et al. Excited-state spectroscopy using single spin manipulation in diamond. Phys. Rev. Lett. 101, 117601 (2008).

158. Alkauskas, A., Buckley, B. B., Awschalom, D. D. \& de Walle, C. G. V. First-principles theory of the luminescence lineshape for the triplet transition in diamond NV centres. New J. Phys. 16, 073026 (2014).

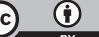

Open Access This article is licensed under a Creative Commons Attribution 4.0 International License, which permits use, sharing, adaptation, distribution and reproduction in any medium or format, as long as you give appropriate credit to the original author(s) and the source, provide a link to the Creative Commons license, and indicate if changes were made. The images or other third party material in this article are included in the article's Creative Commons license, unless indicated otherwise in a credit line to the material. If material is not included in the article's Creative Commons license and your intended use is not permitted by statutory regulation or exceeds the permitted use, you will need to obtain permission directly from the copyright holder. To view a copy of this license, visit http://creativecommons. org/licenses/by/4.0/.

(c) The Author(s) 2018 